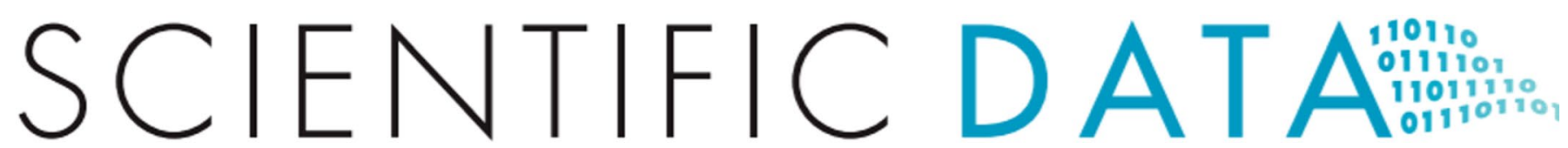

Published online: 15 September 2020

\title{
Author Correction: GlobalFungi, a global database of fungal occurrences from high-throughput- sequencing metabarcoding studies
}

Tomáš Větrovský, Daniel Morais, Petr Kohout, Clémentine Lepinay, Camelia Algora, Sandra Awokunle Hollá, Barbara Doreen Bahnmann, Květa Bílohnědá, Vendula Brabcová, Federica D’Alò, Zander Rainier Human, Mayuko Jomura (D, Miroslav Kolařík, Jana Kvasničková, Salvador Lladó, Rubén López-Mondéjar, Tijana Martinović, Tereza Mašínová, Lenka Meszárošová, Lenka Michalčíková, Tereza Michalová, Sunil Mundra,

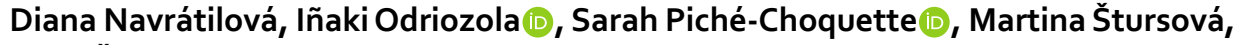
Karel Švec, Vojtěch Tláskal (D, Michaela Urbanová, Lukáš VIk, Jana Vořišková, Lucia Žifčáková \& Petr Baldrian

Correction to: Scientific Data https://doi.org/10.1038/s41597-020-0567-7, published online 13 July 2020

The legend of Fig. 1 has been updated to include the following text acknowledging the source and copyright of the background map image:

"The background map image where the samples are represented is the intellectual property of Esri and used herein under license. Copyright (c) 2019 Esri and its licensors. All rights reserved."

(i) Open Access This article is licensed under a Creative Commons Attribution 4.0 International License, which permits use, sharing, adaptation, distribution and reproduction in any medium or format, as long as you give appropriate credit to the original author(s) and the source, provide a link to the Creative Commons license, and indicate if changes were made. The images or other third party material in this article are included in the article's Creative Commons license, unless indicated otherwise in a credit line to the material. If material is not included in the article's Creative Commons license and your intended use is not permitted by statutory regulation or exceeds the permitted use, you will need to obtain permission directly from the copyright holder. To view a copy of this license, visit http://creativecommons.org/licenses/by/4.0/.

(c) The Author(s) 2020 